\title{
Linagliptin ameliorates hepatic steatosis via non-canonical mechanisms in mice treated with a dual inhibitor of insulin receptor and IGF-1 receptor
}

\author{
Tomoko Okuyama $^{1}$, Jun Shirakawa ${ }^{1}$, Kazuki Tajima ${ }^{1}$, Yoko Ino ${ }^{1}$, Heidrun Vethe ${ }^{2}$, Yu \\ Togashi $^{1}$, Mayu Kyohara ${ }^{1}$, Ryota Inoue ${ }^{1}$, Daisuke Miyashita ${ }^{1}$, Jinghe Li $^{1}$, Nozomi Goto ${ }^{1}$, \\ Taiga Ichikawa ${ }^{1}$, Shingo Yamasaki ${ }^{1}$, Haruka Ohnuma ${ }^{1}$, Rie Takayanagi ${ }^{1}$, Yayoi Kimura ${ }^{1}$, \\ Hisashi Hirano ${ }^{3}$, and Yasuo Terauchi ${ }^{1}$ \\ ${ }^{1}$ Yokohama City University \\ ${ }^{2}$ University of Bergen \\ ${ }^{3}$ Gumma Paz College
}

May 6, 2020

\begin{abstract}
ABSTRACT Background and Purpose: Abnormal hepatic insulin signaling is a cause or consequence of hepatic steatosis. DPP-4 inhibitors might be protective against fatty liver. We previously reported that the systemic inhibition of insulin receptor (IR) and IGF-1 receptor (IGF1R) by the administration of OSI-906 (linsitinib), a dual IR/IGF1R inhibitor, induced glucose intolerance, hepatic steatosis, and lipoatrophy in mice. In the present study, we investigated the effects of a DPP-4 inhibitor, linagliptin, on hepatic steatosis in OSI-906-treated mice. Experimental Approach: We treated C57BL/6J male mice either with vehicle, linagliptin, OSI-906 or OSI-906 + linagliptin for 7 days. We also conducted proteomic and phosphoproteomic analyses of the liver from those mice. Key Results: Unlike high-fat diet-induced hepatic steatosis, OSI-906-induced hepatic steatosis is not characterized by elevations in inflammatory responses or oxidative stress levels. Linagliptin improved OSI-906-induced hepatic steatosis via an insulin-signaling-independent pathway, without altering glucose levels, free fatty acid levels, gluconeogenic gene expressions in the liver, or visceral fat atrophy. Hepatic quantitative proteomic and phosphoproteomic analyses revealed that perilipin-2 (PLIN2), major urinary protein 20 (MUP20), cytochrome P450 2 b10 (CYP2B10), nicotinamide N-methyltransferase (NNMT), and sirtuin families are possibly involved in the process of the amelioration of hepatic steatosis by linagliptin. Conclusion and Implications: Linagliptin improved hepatic steatosis induced by IR and IGF1R inhibition via a previously unknown mechanism that did not involve gluconeogenesis, lipogenesis, or inflammation, suggesting the non-canonical actions of DPP-4 inhibitors in the treatment of hepatic steatosis under insulin-resistant conditions.
\end{abstract}

\section{INTRODUCTION}

The prevalences of patients with non-alcoholic fatty liver disease (NAFLD) and nonalcoholic steatohepatitis (NASH), which are associated with diabetes and metabolic syndrome, have increased considerably (Ahmadieh \& Azar, 2014; Sanyal, 2002). Hepatic insulin action through growth hormone receptors-mediated signaling is involved in the development of fatty liver (Michael et al., 2000). Fat accumulation in the liver has also been found to cause hepatic insulin resistance (Samuel et al., 2004).

Insulin receptor (IR) and IGF-1 receptor (IGF1R) play roles in systemic metabolic actions, cell proliferation and migration, as well as cancer growth and metastasis (Belfiore, Frasca, Pandini, Sciacca \& Vigneri, 2009; Djiogue et al., 2013). Previously, a number of anti-IGF-1 receptor drugs, including monoclonal antibodies and tyrosine kinase inhibitors, have been developed as anti-tumor drugs (Garcia-Echeverria, 2006; Rodon, 
DeSantos, Ferry \& Kurzrock, 2008). OSI-906 (linsitinib) is an orally bioavailable dual IR/IGF1R tyrosine kinase inhibitor (Mulvihill et al., 2009). OSI-906 specifically inhibits the autophosphorylation of IR/IGF1R and their downstream pathways, resulting in the induction of insulin resistance. We previously reported that the oral administration of OSI-906 for 7 days induced glucose intolerance, liver steatosis, and lipoatrophy in mice (Shirakawa et al., 2014; Tajima et al., 2017). In this model, insulin signaling in the liver was completely abolished (Shirakawa et al., 2020).

Linagliptin, a selective dipeptidyl peptidase-4 (DPP-4) inhibitor, is mainly excreted in feces, though most DPP-4 inhibitors are cleared by the kidneys (Blech, Ludwig-Schwellinger, Grafe-Mody, Withopf \& Wagner, 2010). The functions of incretins are thought to potentiate glucose-dependent insulin secretion from pancreatic $\beta$-cells, inhibiting glucagon secretion; this in turn reduces hepatic gluconeogenesis. In addition to their glucose-lowering effects, DPP-4 inhibitors reportedly have multiple pleiotropic effects that are independent of its pancreatic effects. Reportedly, DPP-4 inhibitors might ameliorate NAFLD in patients with type 2 diabetes (Carbone, Angus \& Yeomans, 2016; Samson \& Bajaj, 2013). DPP-4 inhibitors also reduced hepatic fat accumulation in experimental models of NAFLD (Kern et al., 2012; Klein et al., 2014). However, the mechanisms responsible for the protective effects of DPP-4 inhibition on fatty liver are obscure.

In this study, we administered the DPP-4 inhibitor linagliptin to OSI-906-injected mice to investigate whether linagliptin ameliorates fatty liver under the conditions of IR and IGF1R inhibition.

\section{METHODS}

\subsection{Animals and animal care}

C57BL/6J male mice (CLEA Japan, Tokyo, Japan) aged 8 weeks were fed standard chow (Oriental Yeast, Tokyo, Japan) and were allowed free access to food and water at room temperature $\left(25^{\circ} \mathrm{C}\right)$ under a 12 $\mathrm{h}$ light cycle. The mice were randomly divided into four groups, and treated with the vehicle, linagliptin (Lina), OSI-906, or OSI-906 in combination with linagliptin (OSI-906 + Lina) for 7 days. This study was approved by the Yokohama City University Institutional Animal Care and Use Committee (IACUC) (Permit Number: F-A-14-041) and was conducted in accordance with the guidelines of the Animal Care Committee of Yokohama City University.

\subsection{Drugs}

OSI-906 (linsitinib) was purchased from MedChem Express (NJ, USA). Eight-week-old mice were orally administered $10 \mu \mathrm{L} / \mathrm{g}$ body weight of water or linagliptin $(3 \mathrm{mg} / \mathrm{kg} /$ daily, oral gavage) (provided by Boehringer Ingelheim, Ingelheim, Germany) and were also additionally administered $10 \mu \mathrm{L} / \mathrm{g}$ body weight of either the vehicle (30\% Solutol HS-15; BASF, Ludwigshafen am Rhein, Germany) or OSI-906 (45 mg/kg) orally at 30 min after the previous administration once a day for 7 days. Liraglutide was obtained from Novo Nordisk (Bagsvaerd, Denmark). AML-12 cells were treated with $10 \mathrm{nM}$ linagliptin or $100 \mathrm{nM}$ liraglutide in the presence of $200 \mathrm{nM}$ OSI-906.

\subsection{Biochemical parameters}

The plasma glucose levels were determined using Glutest Neo Super (Sanwa Chemical Co. Kanagawa, Japan) just before and 4 hours after the administration of OSI-906 or the vehicle. Serum insulin levels were determined using an insulin kit (Morinaga Institute of Biological Science, Yokohama, Japan). The glycogen content in the liver was determined using a Determiner-GL-E Kit (Wako Pure Chemical Industries). The serum glutamic pyruvic transaminase (GPT), free fatty acid (FFA), and triglyceride (TG) levels were assayed using enzymatic methods (Wako Pure Chemical Industries).

\subsection{Histological analysis}

Formalin-fixed, paraffin-embedded liver and epididymal fat tissue sections were stained with hematoxylin and eosin.

\subsection{Real-time PCR}


Total RNA was isolated from the liver using an RNase free DNase and RNeasy Kit (Qiagen, Valencia, CA). cDNA was prepared using High Capacity cDNA Reverse Transcription Kits (Applied Biosystems) and was subjected to quantitative PCR using THUNDERBIRD SYBR qPCR Mix (Toyobo Co., Ltd., Osaka, Japan). The data were normalized according to the mRNA expression levels of $\beta$-actin and TATA box-binding protein (Tbp). The primer sequences are shown in Supplementary Table 1.

\subsection{Immunoblotting}

For immunoblotting, $40 \mathrm{mg}$ of liver tissues were lysed with T-PER tissue protein extraction reagent (Thermo Fisher Scientific) with protease inhibitor (Nacalai Tesque) and phosphatase inhibitor (Nacalai Tesque). After centrifugation, the extracts were subjected to immunoblotting with antibodies to acetyl-lysine (Abcam \#21623, RRID:AB_446436, 1/1000), perilipin 2 (PROGEN \#42, 1/2000), NNMT (Abcam \#119758, RRID: AB_10902083, 1/600), and glyceraldehyde-3-phosphate dehydrogenase (GAPDH) (Cell Signaling Technology \#5174, RRID: AB_10622025, 1/1000). Densitometry was performed using Image J software.

\subsection{Cell culture}

Mouse hepatocyte AML-12 cells were obtained from ATCC (American Type Culture Collection, Manassas, VA, Cat\# CRL-2254, RRID:CVCL_0140) and were cultured in Dulbecco's modified Eagle's medium/Ham's F-12 (GIBCO) supplemented with $10 \%$ fetal bovine serum (FBS), a mixture of insulin, transferrin, and selenium (ITS; Collaborative Research), and $0.1 \mu \mathrm{M}$ dexamethasone at $37^{\circ} \mathrm{C}$ in a $5 \% \mathrm{CO}_{2}$ atmosphere. Mycoplasma contamination was not detected by 16s rRNA-based mycoplasma group-specific PCR. AML-12 cells were used between passages 15 and 16 .

\subsection{Sample preparation for LC-MS/MS}

The liver was homogenized in lysis buffer containing $50 \mathrm{mM} \mathrm{NH}_{4} \mathrm{HCO}_{3}, 8 \mathrm{M}$ urea, $4 \%$ deoxycholic acid, $1 \%$ phosphatase inhibitor cocktail 2 (SIGMA, St. Louis, MO), 1\% phosphatase inhibitor cocktail 3 (SIGMA, St. Louis, MO), and protease inhibitor cocktail (Roche, Penzberg, Germany). Lysate was obtained after centrifugation at $15000 \mathrm{rpm}$ for $10 \mathrm{~min}$ at $4^{\circ} \mathrm{C}$ and precipitation with $4 \times$ volume of cold acetone, then reconstituted with an appropriate volume of lysis buffer. A total of $110 \mu \mathrm{g}$ of proteins extracted from each liver sample was reduced with dithiothreitol $(10 \mathrm{mM}$ final concentration) and alkylated with iodoacetamide $(25$ $\mathrm{mM}$ final concentration). After dilution with $3 \times$ volume of $50 \mathrm{mM} \mathrm{NH}_{4} \mathrm{HCO}_{3}$, the proteins were digested with trypsin (protein-to-enzyme ratio of 20:1) (Promega) for $18 \mathrm{~h}$ at $37^{\circ} \mathrm{C}$. The protein digests were desalted using C18 StageTips with C18 Empore disks (3 M, St. Paul, MN, USA) following the removal of SDC (Masuda, Tomita \& Ishihama, 2008; Rappsilber, Mann \& Ishihama, 2007). The eluted peptides were used after being completely lyophilized in a vacuum concentrator. For phosphopeptide enrichment, we used Titansphere Phos$\mathrm{TiO}$ beads (GL Sciences, Tokyo, Japan) according to the manufacturer's protocol. Enriched phosphopeptides were desalted using C18 StageTips and lyophilized in a vacuum concentrator.

\subsection{Proteomic and phosphoproteomic analyses}

Total peptides and phosphopeptides were analyzed using LTQ-Orbitrap Elite (Thermo Fisher Scientific Inc. MA, USA) coupled with a Dionex Ultimate 3000 RSLC nano system (Thermo Fisher Scientific) and a QExactive mass spectrometer (Thermo Fisher Scientific Inc.) coupled with a Dionex Ultimate 3000 RSLC nano system, respectively. Label-free quantitation was performed using Progenesis QI for proteomics software (Nonlinear Dynamics, Newcastle, UK). The samples were divided into four groups: vehicle, OSI-906, linagliptin, and OSI-906 + linagliptin. Then, the samples were subjected to separate multivariate statistical analyses of the proteomic and phosphoproteomic data. MS/MS ion searches to identify proteins were performed using MASCOT software (version 2.5.1, Matrix Science, London, UK) against the UniProtKB database (Mus musculus, 16,678 sequences, http://www.uniprot.org/). For total peptides, protein identification was performed using the following parameters: enzyme, trypsin; peptide mass tolerance, $5 \mathrm{ppm}$; fragment mass tolerance, 0.5 Da; maximum missed cleavages, 2; and variable modifications such as protein N-terminal acetylation, carbamidomethylation of cysteine, N-terminal carbamylation and oxidation of methionine. For phosphopeptides, protein identification was performed using the following parameters: enzyme, trypsin; pep- 
tide mass tolerance, 5 ppm; fragment mass tolerance, 0.05 Da; maximum missed cleavages, 2; and variable modifications such as protein N-terminal acetylation, carbamidomethylation of cysteine, N-terminal carbamylation, oxidation of methionine, phosphorylation of serine/threonine and phosphorylation of tyrosine. We used a $1 \%$ overall false discovery rate as a cutoff value to export our results from the database search. In addition, peptides that yielded a peptide ion score of greater than or equal to 30 were used for relative quantitation. Proteins or phosphopeptides with significant quantitative changes were selected according to calculations performed using Progenesis QI for proteomics and the following parameters: analysis of variance (ANOVA) $P$ value $<0.05$, and fold change $>1.2$.

\subsection{IPA functional enrichment analysis}

Genes mapped from significantly upregulated or downregulated peptides and phosphopeptides were used to identify cellular and molecular processes, pathways and upstream regulators using Ingenuity pathway analysis (IPA) software (QIAGEN Redwood City,http://www.qiagen.com/ingenuity). Genes were queried against the Ingenuity knowledge database as the reference set. The regulated proteins, phosphoproteins and their log2transformed SILAC ratios were uploaded into the IPA software, and the top canonical pathways associated with the uploaded phosphoproteins were listed along with the $p$ values calculated using a right-tailed Fisher exact test. Upstream regulators refer to the upstream proteins that are responsible for causing changes in the phosphorylation and/or total expression levels of the queried genes/proteins in the dataset. Activation z-scores were calculated using IPA's z-score algorithm to predict the overall activation or inhibition of the identified functional cellular processes/pathways and upstream regulators. A positive z-score (z-score $>0$ ) implies an overall predicted activation of the process/pathway/upstream regulator, whereas a negative z-score (z-score < 0) implies an overall predicted inhibition or downregulation of the pathway/process/upstream regulator. Z-scores of [?] 2 or [?] -2 were considered by IPA to predict significant activation or inhibition, respectively. Cellular processes/upstream regulators with no z-scores imply that IPA was unable to generate prediction states for these functionalities.

\subsection{Statistical analyses}

Statistical analyses were performed using SPSS statics 19 (IBM). Numbers for every experiment are given in the figure legends and refer to the number of every individual mouse used in the respective group. All the data were expressed as the means +- SEM and were analyzed using ANOVA with an additional Tukey-Kramer post-hoc test. Differences with $P$ values $<0.05\left(^{*}\right)$ or $<0.01(* *)$ were considered significant. Assessment of significance was only done for numbers [?] 5 unless for the animal experiments, when

only some groups had lower sample sizes (Figures 2e and $4 \mathrm{~b}$ ).

\section{RESULTS}

\subsection{Linagliptin reduced plasma triglyceride levels without altering blood glucose and serum insulin levels in OSI-906-treated mice}

In this study, wild-type C57BL/6J mice were orally injected with OSI-906 at a dosage of $45 \mathrm{mg} / \mathrm{day}$, thereby blunting IR and IGF1R-mediated signaling in the liver and white adipose tissue (Shirakawa et al., 2020; Tajima et al., 2017). Linagliptin was administered by oral gavage at a dosage of $3 \mathrm{mg} / \mathrm{kg} / \mathrm{day}$, thereby significantly inhibiting DPP-4 activity and significantly increasing the GLP-1 level (Kern et al., 2012). The mice were treated with the vehicle, linagliptin (Lina), OSI-906, or OSI-906 in combination with linagliptin (OSI-906 + Lina) for 7 days (Figure 1a). The oral administration of OSI-906 transiently decreased the body weight gain observed on day 3 (Figure 1b). Linagliptin prevented the OSI-906-induced reductions in body weight gain observed on day 3 (Figure 1a). Blood glucose levels became significantly elevated at 4 hours after OSI-906 administration, and linagliptin partially reduced the blood glucose levels observed on days 1 and 2 (Figure 1c). However, hyperglycemia elicited by OSI-906 administration did not improve with linagliptin treatment after day 3 (Figure 1c).

On day 7 , the serum insulin levels became significantly higher after OSI-906 administration, consistent with the inhibition of IR/IGF1R. Treatment with linagliptin did not influence the hyperinsulinemia observed 
in mice treated with OSI-906 (Figure 1d). On the other hand, linagliptin canceled the OSI-906-induced elevation in plasma triglyceride levels, although no significant differences in plasma free fatty acid (FFA) levels were observed between the OSI-906 and OSI-906 + Lina groups (Figure 1e and F). The serum glutamic pyruvic transaminase (GPT) levels were not altered by the administration of OSI-906 or by treatment with linagliptin (Figure 1g).

\subsection{Linagliptin improved OSI-906-induced hepatic steatosis}

We previously reported that OSI-906 administration induced lipoatrophy and hepatic steatosis after 7 days of administration in wild-type mice (Tajima et al., 2017). We also reported that DPP-4 inhibition prevented diet-induced adipose tissue inflammation and hepatic steatosis in diabetic mice (Shirakawa et al., 2016). Next, we investigated the impact of DPP-4 inhibition on lipoatrophy or hepatic steatosis in wild-type mice treated with OSI-906 for 7 days. The atrophic changes in visceral fat elicited by OSI-906 were not affected by the treatment with linagliptin (Supplementary Figure 1a and b). In contrast, DPP-4 inhibition with linagliptin improved OSI-906-induced hepatic steatosis (Figure 2a). Thus, we further assessed the effects of linagliptin on the liver in OSI-906 treated mice. The administration of OSI-906 significantly increased the liver weight, and this increase in liver weight was significantly lower in the OSI-906 + Lina group than in the OSI-906 group (Figure 2b). The hepatic triglyceride content and the hepatic glycogen content were significantly increased in the OSI-906 group, whereas these parameters were reversed by the treatment with linagliptin (Figure 2c and d).

We next examined the hepatic gene expression involved in hepatic metabolism on day 7 (Figure 2e). The expressions of gluconeogenic genes, such as G6pase and Pepck, and a potent activator of gluconeogenesis, $\Pi \gamma \mathcal{S}_{-}$-1a, were increased in the liver by the OSI-906 administration, consistent with the impairment of hepatic insulin action caused by the blocking of IR and IGF1R. The treatment with linagliptin did not change these expressions. The expressions ofFas and $S c d 1$, which are involved in de novo lipogenesis, were not altered in the presence of linagliptin in OSI-906-treated mice. We also investigated the expressions of genes related to inflammation and oxidative stress in the liver because these processes are closely related to the development of hepatic steatosis or NAFLD (Figure 2e). Interestingly, the expressions of inflammatory genes, such as Tnfa, Il-6, Ccl2, and $i N o s$, tended to be reduced by the administration with OSI-906 for 7 days. Linagliptin restored the reductions in the expressions of Tnf-a, Il-6, and Ccl2 in the liver. In addition, PAI-1 expression tended to be reduced by linagliptin treatment in both OSI-906-treated and untreated groups.

\subsection{Hepatic proteomic and phosphoproteomic analyses revealed insulin- and glucose- inde- pendent effects of linagliptin in OSI-906-induced hepatic steatosis}

We also performed hepatic quantitative proteomic and phosphoproteomic analyses of the livers from OSI-906or linagliptin-treated mice (Figure 3a). We identified a total of 1884 proteins, and differentially expressed proteins were analyzed using Progenesis QI software to compare expressions in the OSI-906 vs. vehicle groups and the OSI-906 + Lina vs. OSI-906 groups (Figure 3b).

We focused on proteins with expressions that were significantly altered by the administration of OSI-906 and for which the alterations were reversed by the treatment with linagliptin (Figure 3c and Supplementary Table 2 ) to address the amelioration of hepatic steatosis by linagliptin in the OSI-906-treated model. Among the proteins that were significantly up-regulated or down-regulated in response to the administration of OSI-906 (Supplementary Tables 2-4), the abundances of perilipin-2 (PLIN2) and cytochrome P450 2b10 (CYP2B10) were reduced by OSI-906 and were restored by treatment with linagliptin. In contrast, the abundance of major urinary protein 20 (MUP20) was increased by OSI-906 and was also restored by treatment with linagliptin. Those results indicate that these molecules might contribute to the process of the linagliptininduced amelioration of hepatic steatosis. A previous study reported that the expressions of cytochrome P450 enzymes were down-regulated and those of major urinary proteins (MUPs) were up-regulated by treatment with resveratrol, a sirtuin activator, in the liver (Baur et al., 2006). We also found that the protein expression of nicotinamide N-methyltransferase (NNMT) was significantly up-regulated in OSI-906-

treated liver. Recently, the contribution of NNMT activation to the development of hepatic steatosis has 
been reported (Komatsu et al., 2018). Therefore, these findings suggest that sirtuin activity is regulated by OSI-906 administration and might contribute to the improvement in hepatic steatosis (Pissios, 2017). We validated these findings using immunoblotting or gene expression analyses. The protein expressions of PLIN2 and NNMT were increased in OSI-906-treated liver, and these increases were reversed by linagliptin (Figure 4a). In addition, the increased pan acetyl-lysine levels in OSI-906-treated liver were also reversed by linagliptin, implying an enhancement of sirtuin activity by linagliptin in this OSI-906-induced hepatic steatosis model (Figure 4a). The hepatic gene expression of Cyp2b10 was also induced by OSI-906 and was deceased by treatment with linagliptin (Figure 4b). A canonical pathway analysis of differentially expressed proteins in the OSI-906 vs. vehicle groups and the OSI-906 + Lina vs. OSI-906 groups revealed that the administration of OSI-906 activated the cholesterol biosynthesis pathway and inhibited the LXR/RXR pathway, whereas linagliptin inhibited the cholesterol biosynthesis pathway (Figure 5).

In a phosphoproteomic analysis of liver samples from the four groups, more than 3700 sites of phosphorylation were identified. The top increased or decreased phosphopeptides when the OSI-906 vs. vehicle groups or the OSI-906 + Lina vs. OSI-906 groups were compared are shown in Supplementary Tables 5-8.

A canonical pathway analysis of the phosphoproteomics data identified the inhibition of insulin receptor signaling and mTOR signaling in OSI-906-treated liver, consistent with the dual inhibition of insulin/IGF1 receptors by OSI-906 (Figure 6). Treatment with linagliptin did not seem to affect insulin signaling. An upstream regulator analysis indicated that PPAR $\alpha$ and XBP1 were the top 2 predicted transcription activators involved in the process of hepatic steatosis induced by OSI-906, whereas pathways related to SREBF2 and lysophosphatidylcholine were identified in the linagliptin-treated group (Supplementary Table $9)$.

\subsection{Linagliptin exerts direct and indirect effects on hepatocytes during the improvement of hepatic steatosis}

To elucidate whether the effect of linagliptin on hepatic steatosis was mediated through the direct action of linagliptin, GLP-1 receptor signaling in hepatocytes, or an indirect pathway through components such as humoral factors, we treated hepatocyte AML-12 cells with the DPP-4 inhibitor linagliptin or the GLP-1 receptor agonist liraglutide in the presence of OSI-906 (Figure 7). In AML-12 hepatocyte cells, linagliptin did not reverse the effects of OSI-906 on gluconeogenic gene expressions, consistent with the in vivo results. In contrast, the decrease in Tnf expression in OSI-906-treated cells was not reversed by incubation with linagliptin or liraglutide, unlike thein vivo results, implying an indirect effect of linagliptin and DPP-4 inhibition on hepatocytes. In contrast, liraglutide canceled the OSI-906-induced increases in gluconeogenic gene expressions. The expression of Plin2 was induced by OSI-906 and was diminished by the treatment with linagliptin, but not with liraglutide. These results indicate that linagliptin influences hepatocytes through multiple mechanisms that are independent of GLP-1 receptor signaling.

\section{DISCUSSION}

In this study, we reported that the DPP-4 inhibitor linagliptin ameliorated hepatic steatosis elicited by the dual IR/IGF1R inhibitor OSI-906. We previously reported that a DPP-4 inhibitor, des-fluoro-sitagliptin, prevented diet-induced adipose tissue inflammation and hepatic steatosis (Shirakawa et al., 2011b). In another study, linagliptin reportedly improved insulin sensitivity and hepatic steatosis in diet-induced obese (DIO) mice (Kern et al., 2012). DPP-4 inhibition with linagliptin has been shown to decrease the expressions of SREBP1c, SCD-1 and FAS, all of which are known to be related tode novo lipogenesis, and increased the expression of PPAR $\alpha$ in the livers of DIO mice (Kern et al., 2012). On the other hand, the expressions of genes involved in hepatic fatty acid oxidation were not affected by treatment with linagliptin in DIO or NASH mouse models (Kern et al., 2012; Klein et al., 2014). However, the mechanism by which DPP-4 inhibitors improve fatty liver in the presence of diet-induced obesity or diabetes remains unclear.

IR and IGF-1R-mediated signaling play a crucial role in hepatic insulin action, which regulates glucose and fatty acid metabolism. OSI-906 administration induces the acute inhibition of IR/IGF1R signaling and evokes hepatic steatosis (Tajima et al., 2017). In our mouse model, the dual inhibition of IR and 
IGF-1R with OSI-906 resulted in impaired insulin signaling in the liver. The liver weight and liver TG contents were significantly increased by the administration of OSI-906 for 7 days. Linagliptin significantly reversed the OSI-906-induced increase in liver weight and TG content. The OSI-906 group exhibited increased gluconeogenic gene expression, compared with the vehicle group, and treatment with linagliptin did not affect the expressions of these genes, consistent with an impairment in hepatic insulin action. The expressions of Gck, Srebp1c, Fas, and ППАPa, which were also regulated by insulin signaling, showed no significant alteration by treatment with linagliptin in OSI-906 treated mice. Hence, the effect of linagliptin on hepatic steatosis seemed to be independent of the IR/IGF1R signaling pathway. Our data indicated that although insulin signaling is involved in the development of hepatic steatosis, an alternative pathway that improves liver steatosis without altering insulin signals likely exists in the OSI-906-treated model. The OSI-906 + Lina group had lower glucose levels at 4 hours after OSI-906 administration on days 1 and 2, compared with the levels in the OSI-906 group, but these effects were abolished after day 3. Hyperinsulinemia evoked by OSI906 was also observed in the OSI-906 + Lina group. Therefore, the glucose-lowering effects of linagliptin did not contribute to the improvement in OSI-906-induced hepatic steatosis. In patients with metabolic syndrome, fatty liver occurs because of an increased uptake of fatty acids accompanied by an impairment in insulin action in adipose tissue. In this study, linagliptin did not reduce the plasma free fatty acid levels and hepatic expression of $C d 36$, which is a free fatty acid transporter, in OSI-906-treated mice. Therefore, linagliptin seemed to ameliorate OSI-906-induced hepatic steatosis independently of the fatty acid flux to the liver, consistent with the regulation of fatty acid metabolism via insulin. Thus, linagliptin improved OSI-906-induced hepatic steatosis via a pathway that was independent of insulin signaling, glucose levels, or free fatty acid metabolism.

Liver-specific IR knockout (LIRKO) mice showed severe insulin resistance and glucose intolerance with increased hepatic glucose production, impaired glucose utilization in the liver, and abnormal mitochondrial function because of the failure of insulin activity (Cheng et al., 2009; Michael et al., 2000; Titchenell, Chu, Monks \& Birnbaum, 2015). The loss of FoxO1 increases lipogenesis and decreases fatty acid oxidation in hepatocytes. Thus, liver FoxO1-null mice developed hepatic steatosis, accompanied by the upregulation of lipogenic genes. This regulation of lipogenesis by FoxO1 requires intact hepatic insulin signaling. The systemic inhibition of IR with S961 blunted the effects of insulin on hepatic glucose production in liver-specific IR/FoxO1 double knockout LIRFKO mice (I et al., 2015). Thus, systemic insulin signaling might be required to regulate hepatic glucose production and de novolipogenesis. On the other hand, lipodystrophy in adipose tissue-specific IR knockout (F-IRKO) mice causes progressive NAFLD (Softic et al., 2016). Treatment with OSI-906 also induced lipodystrophy and hepatic steatosis, similar to the F-IRKO mouse model. These findings indicate that not only hepatic insulin signaling, but also insulin signaling in adipose tissue plays a crucial role in the development in hepatic steatosis. However, in this study, linagliptin did not reverse the lipodystrophy induced by OSI-906 administration, implying that linagliptin exerts its effect on OSI906-induced hepatic steatosis without altering either hepatic insulin signaling or insulin signaling in adipose tissue.

Chronic low-grade inflammation is known to contribute to the development of steatosis (Tilg \& Moschen, 2010). Interestingly, the inhibition of IR and IGF1R by OSI-906 showed a tendency to decrease inflammatory gene expressions. These results indicate that OSI-906-induced hepatic steatosis is not provoked by the inflammatory response, unlike high-fat diet-induced hepatic steatosis. We previously reported that DPP-4 inhibition prevented diet-induced hepatic steatosis and adipose tissue inflammation in a diabetic mouse model (Shirakawa et al., 2011a). We also showed that the PAI-1 expression levels in adipose tissue were elevated in diet-induced hepatic steatosis (Shirakawa et al., 2011a). In the present study, PAI-1 expression in the liver tended to decrease with linagliptin treatment in the absence or presence of OSI-906, though a significant difference was not observed. Linagliptin reportedly reduced advanced glycation end products (AGE)-related oxidative stress in the kidneys of a type 1 diabetes mouse model (Nakashima, Matsui, Takeuchi \& Yamagishi, 2014), whereas no significant differences in the expressions of genes related to oxidative stress were observed in the current study. Thus, in this OSI-906-induced hepatic steatosis model, the identification of a new molecular mechanism for hepatic steatosis via insulin resistance, other than inflammation or oxidative stress, 
might be useful for understanding the development of hepatic steatosis.

Proteomic and phosphoproteomic analyses revealed some possible mechanisms underlying OSI-906-induced progression in hepatic steatosis and its amelioration by treatment with linagliptin. We focused on molecules such as PLIN2 and sirtuins, which were suggested by a proteomic analysis to be responsible for the effect of linagliptin on hepatic steatosis. PLIN2, a lipid droplet-coating protein, is related to lipid accumulation in the liver and promotes hepatic steatosis (Hall et al., 2010; Libby, Bales, Orlicky \& McManaman, 2016; Najt et al., 2016). PPAR $\gamma$ is also known to be required for the induction of PLIN2. In a quantitative proteomic analysis, OSI-906 administration significantly up-regulated PLIN2, whereas treatment with linagliptin almost canceled the OSI-906-induced elevation in PLIN2. These results imply that the regulation of PLIN2 in the liver is associated with the development of hepatic steatosis induced by OSI-906 and its recovery as a result of DPP-4 inhibition using linagliptin. Similarly, CYP2B10 was up-regulated in OSI-906-treated liver, and treatment with linagliptin reversed this induction. Regarding MUP20, its expression was reduced by OSI906 and restored by linagliptin. The regulation of Cyp2b10 and Mup20 were consistent with the context reported for high-fat-diet-induced steatosis and its recovery by treatment with resveratrol (Baur et al., 2006). Cyp2b10 is related to lipid metabolism in the liver, and one of the regulators of Cyp2b10 is a constitutive androgen receptor (CAR) (Ohno, Kanayama, Moore, Ray \& Negishi, 2014). Major urinary proteins (MUPs), which are secreted proteins, belong to the lipocalin family and are predominantly produced by the liver. A previous report showed that MUP1 increases energy expenditure and improves glucose tolerance (Zhou, Jiang \& Rui, 2009). Since resveratrol acts as a sirtuin activator, these data suggest that treatment with linagliptin improves OSI-906-induced hepatic steatosis by regulating sirtuin activity and its upstream and downstream metabolic pathways.

Sirtuin, a nutrient sensor, activates AMPK and downstream fatty acid oxidation in the liver. In fact, NNMT was significantly up-regulated in OSI-906-treated liver in a quantitative proteomic analysis, supporting the impact of sirtuin-mediated metabolic regulation in these mice. Treatment of a human hepatocyte cell line with exendin-4 reportedly increased the transcript levels of sirtuin1 (SIRT1) (Lee et al., 2012). There are several signal transduction pathways that have been proposed to improve hepatic steatosis as a result of incretin-based therapy, such as cAMP-PKA signaling, PI3K-PDK1-Akt/PKB signaling, and AMPK signaling (Samson \& Bajaj, 2013). Whether incretin exerts direct effects or facilitates indirect pathways affecting hepatocyte metabolism remains controversial. Moreover, a recent report showed the importance of hepatocyte-derived DPP-4 and obesity on adipose inflammation and insulin resistance (24).

To address the conflicts in the actions of DPP-4 inhibitors and the GLP-1 receptor agonist on the liver, we examined the effect of linagliptin and the GLP-1 analog liraglutide on hepatocyte AML-12 cells. Linagliptin did not inhibit gluconeogenic gene expressions in OSI-906-treated AML-12 cells, consistent with an insulin signaling-independent action of linagliptin on hepatic steatosis. On the other hand, liraglutide showed a tendency to reduce the expressions of gluconeogenic genes in OSI-906-treated cells. These results suggest that the DPP-4 inhibitor and the GLP-1 analog had different effects on insulin signaling in hepatocytes. In addition, the influence of linagliptin on Tnf expression differed between in vivo andin vitro studies, implying an indirect action of linagliptin on hepatocytes that does not involve the regulation of DPP-4 enzymatic activity.

Taken together, these results show an effect of DPP-4 inhibition on hepatic steatosis that is induced by the acute inhibition of IR/IGF1R signaling through an insulin signaling-independent pathway (Figure 8). Our findings support the non-canonical pleiotropic effects of DPP-4 inhibitors without a glucose-lowering effect and suggest the potential of DPP-4 inhibitors as a new treatment for NAFLD. Further investigation of the underlying mechanism is required.

\section{AUTHOR CONTRIBUTION}

T.O., J.S., and Y.Te. designed the study. T.O., K.T., T.I., S.Y., and J.S. performed the experiments, analyzed the data and wrote the manuscript. Y.I., Y.To, M.K., R.I., D.M., J.L., N.G., H.O.,R.T, Y.K., and H.H. assisted in the experiments. All authors gave final approval of the version to be published. J.S. is the 
guarantor of this work and, as such, had full access to all the data in the study and takes responsibility for the integrity of the data and the accuracy of the data analysis.

\section{ACKNOWLEDGMENT}

We thank Mitsuyo Kaji and Eri Sakamoto (Yokohama City University) for technical assistance, and Misa Katayama for secretarial assistance. The authors thank Boehringer Ingelheim (Ingelheim, Germany) for providing the linagliptin.

\section{FUNDING}

This work was supported in part by Grants-in-Aid for Scientific Research (B) 16H05329 from the Ministry of Education, Culture, Sports, Science and Technology (MEXT) of Japan (to Y.Te.), a Grant-in-Aid for Young Scientists (B) 18K16240 from MEXT of Japan, Junior Scientist Development Grant provided by Novo Nordisk Pharma Ltd., Kanae Foundation for the Promotion of Medical Science, Suzuken Memorial Foundation, Japan Foundation for Applied Enzymology (FFDR), Ono Medical Research Foundation, Kamome Memorial Foundation of Yokohama City University, Takeda Science Foundation, the Mochida Memorial Foundation for Medical and Pharmaceutical Research, and a grant from the Japan IDDM network (to J.S.) and a Grant-in-Aid for Young Scientists (B) from MEXT of Japan, Junior Scientist Development Grant provided by Novo Nordisk Pharma, MSD Life Science Foundation, Japan Diabetes Foundation, Japan Foundatio18K16241n for Applied Enzymology (FFDR), Wakaba Grant provided by Yokohama Foundation for Advancement of Medical Science (to T.O).

\section{CONFLICT OF INTEREST}

No potential conflicts of interest relevant to this article were reported.

\section{DECLARATION OF TRANSPARENCY AND SCIENTIFIC RIGOUR}

This Declaration acknowledges that this paper adheres to the principles for transparent reporting and scientific rigour of preclinical research as stated in the BJP guidelines for

Design \& Analysis, Immunoblotting and Immunochemistry and Animal Experimentation,

and as recommended by funding agencies, publishers and other organisations engaged with supporting research.

\section{REFERENCES}

Ahmadieh H, \& Azar ST (2014). Liver disease and diabetes: association, pathophysiology, and management. Diabetes Res Clin Pract 104:53-62.

Baur JA, Pearson KJ, Price NL, Jamieson HA, Lerin C, Kalra A, et al. (2006). Resveratrol improves health and survival of mice on a high-calorie diet. Nature 444: 337-342.

Belfiore A, Frasca F, Pandini G, Sciacca L, \& Vigneri R (2009). Insulin receptor isoforms and insulin receptor/insulin-like growth factor receptor hybrids in physiology and disease. Endocr Rev 30:586-623.

Blech S, Ludwig-Schwellinger E, Grafe-Mody EU, Withopf B, \& Wagner K (2010). The metabolism and disposition of the oral dipeptidyl peptidase-4 inhibitor, linagliptin, in humans. Drug Metab Dispos 38: 667-678.

Carbone LJ, Angus PW, \& Yeomans ND (2016). Incretin-based therapies for the treatment of non-alcoholic fatty liver disease: A systematic review and meta-analysis. J Gastroenterol Hepatol 31: 23-31.

Cheng Z, Guo S, Copps K, Dong X, Kollipara R, Rodgers JT, et al.(2009). Foxo1 integrates insulin signaling with mitochondrial function in the liver. Nat Med 15: 1307-1311.

Djiogue S, Nwabo Kamdje AH, Vecchio L, Kipanyula MJ, Farahna M, Aldebasi Y, et al. (2013). Insulin resistance and cancer: the role of insulin and IGFs. Endocr Relat Cancer 20: R1-r17. 
Garcia-Echeverria C (2006). Medicinal chemistry approaches to target the kinase activity of IGF-1R. IDrugs 9: 415-419.

Hall AM, Brunt EM, Chen Z, Viswakarma N, Reddy JK, Wolins NE, et al. (2010). Dynamic and differential regulation of proteins that coat lipid droplets in fatty liver dystrophic mice. J Lipid Res 51:554-563.

I OS, Zhang W, Wasserman DH, Liew CW, Liu J, Paik J, et al.(2015). FoxO1 integrates direct and indirect effects of insulin on hepatic glucose production and glucose utilization. Nat Commun 6: 7079 .

Kern M, Kloting N, Niessen HG, Thomas L, Stiller D, Mark M, et al. (2012). Linagliptin improves insulin sensitivity and hepatic steatosis in diet-induced obesity. PloS one 7: e38744.

Klein T, Fujii M, Sandel J, Shibazaki Y, Wakamatsu K, Mark M, et al. (2014). Linagliptin alleviates hepatic steatosis and inflammation in a mouse model of non-alcoholic steatohepatitis. Med Mol Morphol 47: 137-149.

Komatsu M, Kanda T, Urai H, Kurokochi A, Kitahama R, Shigaki S, et al. (2018). NNMT activation can contribute to the development of fatty liver disease by modulating the NAD (+) metabolism. Sci Rep $8: 8637$.

Lee J, Hong SW, Chae SW, Kim DH, Choi JH, Bae JC, et al. (2012). Exendin-4 improves steatohepatitis by increasing Sirt1 expression in high-fat diet-induced obese C57BL/6J mice. PloS one 7: e31394.

Libby AE, Bales E, Orlicky DJ, \& McManaman JL (2016). Perilipin-2 Deletion Impairs Hepatic Lipid Accumulation by Interfering with Sterol Regulatory Element-binding Protein (SREBP) Activation and Altering the Hepatic Lipidome. J Biol Chem 291: 24231-24246.

Masuda T, Tomita M, \& Ishihama Y (2008). Phase transfer surfactant-aided trypsin digestion for membrane proteome analysis. Journal of proteome research 7: 731-740.

Michael MD, Kulkarni RN, Postic C, Previs SF, Shulman GI, Magnuson MA, et al. (2000). Loss of insulin signaling in hepatocytes leads to severe insulin resistance and progressive hepatic dysfunction. Mol Cell 6: 87-97.

Mulvihill MJ, Cooke A, Rosenfeld-Franklin M, Buck E, Foreman K, Landfair D, et al. (2009). Discovery of OSI-906: a selective and orally efficacious dual inhibitor of the IGF-1 receptor and insulin receptor. Future Med Chem 1: 1153-1171.

Najt CP, Senthivinayagam S, Aljazi MB, Fader KA, Olenic SD, Brock JR, et al. (2016). Liver-specific loss of Perilipin 2 alleviates diet-induced hepatic steatosis, inflammation, and fibrosis. Am J Physiol Gastrointest Liver Physiol 310: G726-738.

Nakashima S, Matsui T, Takeuchi M, \& Yamagishi SI (2014). Linagliptin blocks renal damage in type 1 diabetic rats by suppressing advanced glycation end products-receptor axis. Horm Metab Res 46:717-721.

Ohno M, Kanayama T, Moore R, Ray M, \& Negishi M (2014). The roles of co-chaperone CCRP/DNAJC7 in Cyp2b10 gene activation and steatosis development in mouse livers. PloS one 9: e115663.

Pissios P (2017). Nicotinamide N-Methyltransferase: More Than a Vitamin B3 Clearance Enzyme. Trends Endocrinol Metab 28: 340-353.

Rappsilber J, Mann M, \& Ishihama Y (2007). Protocol for micro-purification, enrichment, pre-fractionation and storage of peptides for proteomics using StageTips. Nature protocols 2:1896-1906.

Rodon J, DeSantos V, Ferry RJ, Jr., \& Kurzrock R (2008). Early drug development of inhibitors of the insulin-like growth factor-I receptor pathway: lessons from the first clinical trials. Mol Cancer Ther 7: 2575-2588.

Samson SL, \& Bajaj M (2013). Potential of incretin-based therapies for non-alcoholic fatty liver disease. J Diabetes Complications 27:401-406. 
Samuel VT, Liu ZX, Qu X, Elder BD, Bilz S, Befroy D, et al.(2004). Mechanism of hepatic insulin resistance in non-alcoholic fatty liver disease. J Biol Chem 279: 32345-32353.

Sanyal AJ (2002). AGA technical review on nonalcoholic fatty liver disease. Gastroenterology 123: 17051725 .

Shirakawa J, Amo K, Ohminami H, Orime K, Togashi Y, Ito Y, et al.(2011a). Protective effects of dipeptidyl peptidase-4 (DPP-4) inhibitor against increased beta cell apoptosis induced by dietary sucrose and linoleic acid in mice with diabetes. J Biol Chem 286:25467-25476.

Shirakawa J, Fujii H, Ohnuma K, Sato K, Ito Y, Kaji M, et al.(2011b). Diet-induced adipose tissue inflammation and liver steatosis are prevented by DPP-4 inhibition in diabetic mice. Diabetes 60: 1246-1257.

Shirakawa J, Okuyama T, Kyohara M, Yoshida E, Togashi Y, Tajima K, et al. (2016). DPP-4 inhibition improves early mortality, beta cell function, and adipose tissue inflammation in $\mathrm{db} / \mathrm{db}$ mice fed a diet containing sucrose and linoleic acid. Diabetol Metab Syndr 8:16.

Shirakawa J, Okuyama T, Yoshida E, Shimizu M, Horigome Y, Tuno T, et al. (2014). Effects of the antitumor drug OSI-906, a dual inhibitor of IGF-1 receptor and insulin receptor, on the glycemic control, beta-cell functions, and beta-cell proliferation in male mice. Endocrinology 155: 2102-2111.

Shirakawa J, Tajima K, Okuyama T, Kyohara M, Togashi Y, De Jesus DF, et al. (2020). Luseogliflozin increases beta cell proliferation through humoral factors that activate an insulin receptor- and IGF-1 receptorindependent pathway. Diabetologia 63:577-587.

Softic S, Boucher J, Solheim MH, Fujisaka S, Haering MF, Homan EP, et al. (2016). Lipodystrophy Due to Adipose Tissue-Specific Insulin Receptor Knockout Results in Progressive NAFLD. Diabetes 65:2187-2200.

Tajima K, Shirakawa J, Togashi Y, Yamazaki S, Okuyama T, Kyohara M, et al. (2017). Metabolic recovery of lipodystrophy, liver steatosis, and pancreatic beta cell proliferation after the withdrawal of OSI-906. Sci Rep 7: 4119.

Tilg H, \& Moschen AR (2010). Evolution of inflammation in nonalcoholic fatty liver disease: the multiple parallel hits hypothesis. Hepatology 52: 1836-1846.

Titchenell PM, Chu Q, Monks BR, \& Birnbaum MJ (2015). Hepatic insulin signalling is dispensable for suppression of glucose output by insulin in vivo. Nat Commun 6: 7078.

Zhou Y, Jiang L, \& Rui L (2009). Identification of MUP1 as a regulator for glucose and lipid metabolism in mice. J Biol Chem 284:11152-11159.

\section{FIGURE LEGEND}

Figure 1. Linagliptin improved OSI-906-induced hypertriglyceridemia in mice after 7 days of OSI-906 administration.

(a ) Experimental protocol $(\mathrm{n}=6$ in Vehicle, Lina, OSI-906 + Lina group, respectively, $\mathrm{n}=5$ in OSI-906 group).

(b ) Body weight gain during the experiment (left), on day 3 (middle), and change in body weight on day 7 (right). Data represent the mean \pm SEM. ${ }^{*} P<0.05(\mathrm{n}=6$ in Vehicle, Lina, OSI-906 + Lina group, respectively, $\mathrm{n}=5$ in OSI-906 group).

(c ) Blood glucose levels during the experiment (left) and on day 1 (right).

(d ) Serum insulin, (e ) serum triglyceride (TG), (f ) serum free fatty acid (FFA), and (g ) serum glutamic pyruvic transaminase (GPT) levels on day 7 . 
Data represent the mean \pm SEM. ${ }^{*} P<0.05,{ }^{* *} P<0.01(\mathrm{n}=6$ in Vehicle, Lina, OSI-906 + Lina group, respectively, $\mathrm{n}=5$ in OSI-906 group) by ANOVA with an additional Tukey-Kramer post-hoc test.

Figure 2. Linagliptin improved hepatic steatosis evoked by OSI-906.

(a ) Hematoxylin and eosin-stained sections of liver on day 7. Scale bar $=200 \mu \mathrm{m}$.

(b) Ratio of liver weight to body weight on day 7 ( $\mathrm{n}=6$ in Vehicle, Lina, OSI-906 + Lina group, respectively, $\mathrm{n}=5$ in OSI-906 group).

(c ) (d ) Triglyceride (TG) and glycogen content in the liver on day 7 ( $\mathrm{n}=5$ per group, respectively).

(e ) Hepatic gene expressions of the indicated molecules on day 7 ( $\mathrm{n}=5$ in Vehicle, Lina, OSI-906 + Lina group, respectively, $\mathrm{n}=4$ in OSI-906 group).

Data represent the mean \pm SEM. ${ }^{*} P<0.05,{ }^{* *} P<0.01$ by ANOVA with an additional Tukey-Kramer post-hoc test.

Figure 3. Proteomic and phosphoproteomic analysis of the liver from OSI-906- and linagliptintreated mice.

(a ) Protocol for proteomic and phosphoproteomic analyses using liver samples ( $\mathrm{n}=5$ per group, respectively).

(b ) Volcano plot of hepatic proteomic data. Molecules identified in comparisons of vehicle vs. OSI-906 (left) and OSI-906 vs. OSI-906 + linagliptin are shown ( $\mathrm{n}=5$ per group, respectively).

(c ) Venn diagram of differentially expressed molecules in OSI-906 vs. Vehicle and OSI-906 + Lina vs. OSI906 in proteomic analyses of liver samples. The numbers of proteins that were significantly upregulated or downregulated (ANOVA $P$ value $<0.05$, fold change $>1.2$ ) compared with the respective controls are shown $(\mathrm{n}=5$ per group, respectively). The lists of overlapping proteins are shown in Supplementary Table 1.

Figure 4. Linagliptin reversed the increased expression of acetylated lysine, perilipin-2 and NNMT in OSI-906-treated liver.

(a ) Immunoblotting for pan acetyl-lysine, Perilipin-2, and NNMT in the liver ( $\mathrm{n}=3$ per group, respectively).

(b ) Hepatic gene expressions of Cyp2b10, Plin2and NNMT on day 7. Data represent the mean \pm SEM. ${ }^{*} P<0.05,{ }^{* *} P<0.01(\mathrm{n}=5$ in Vehicle, Lina, OSI-906 + Lina group, respectively, $\mathrm{n}=4$ in OSI-906 group) by ANOVA with an additional Tukey-Kramer post-hoc test.

Figure 5. Canonical pathway analysis of proteomics in the livers of OSI-906- and linagliptintreated mice.

A canonical pathway analysis of the hepatic proteomic data was performed using molecules that were differentially expressed (ANOVA $P<0.05$ ) in comparisons of OSI-906 vs. Vehicle and OSI-906 + Lina vs. OSI-906. The color of the bar indicates the z-score (orange, positive z-score; blue, negative z-score).

Figure 6. Canonical pathway analysis of phosphoproteomics in the livers of OSI-906- and linagliptin-treated mice.

A canonical pathway analysis of the hepatic phosphoproteomic data was performed using molecules that were differentially expressed (ANOVAP $<0.05$ ) in comparisons of OSI-906 vs. Vehicle or OSI-906 + Lina vs. OSI-906. The color of the bar indicates the z-score (orange, positive z-score; blue, negative z-score)

Figure 7. Impact of linagliptin or liraglutide on gene expressions in OSI-906-treated AML-12 cells.

The gene expressions of the indicated genes in AML-12 cells treated with $10 \mathrm{nM}$ linagliptin or $100 \mathrm{nM}$ liraglutide in the presence of $200 \mathrm{nM}$ OSI-906 are shown. Cells were serum-starved overnight and then 
incubated with OSI-906 for 4 hours before the treatment with linagliptin or liraglutide. Then, the cells were incubated for 24 hours with the indicated drugs.

Data represent the mean \pm SEM $(n=4$ in Vehicle, OSI-906 + Linagliptin, OSI-906 + Liraglutide group, respectively, $\mathrm{n}=5$ in OSI-906 group).

Figure 8. Schematic regulatory pathway of linagliptin in OSI-906-induced hepatic steatosis.

Linagliptin improved hepatic steatosis induced by acute IR/IGF1R signaling inhibition with OSI-906 through an insulin signaling-independent pathway. Pathways involving perilipin-2, NNMT, and sirtuins have been proposed as possible mechanisms for the amelioration of OSI-906-induced hepatic steatosis by linagliptin.

Fig. 1

(a)

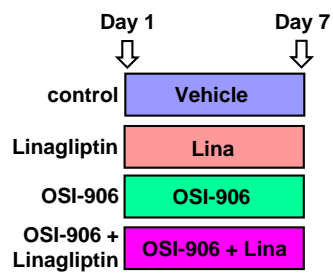

(b)
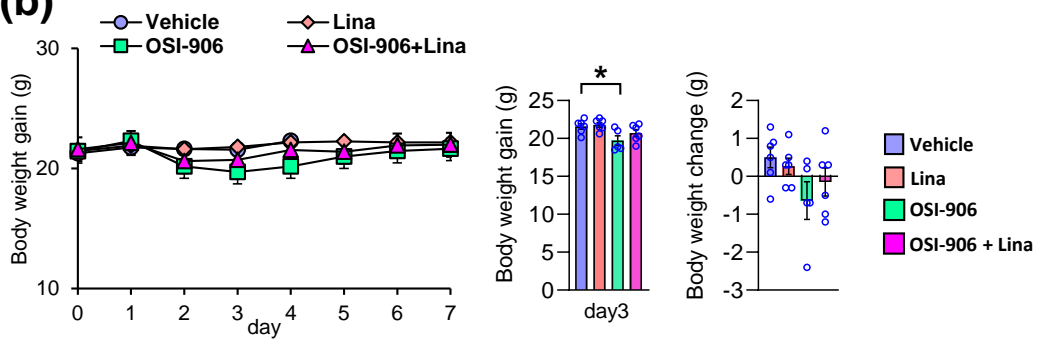

(c)
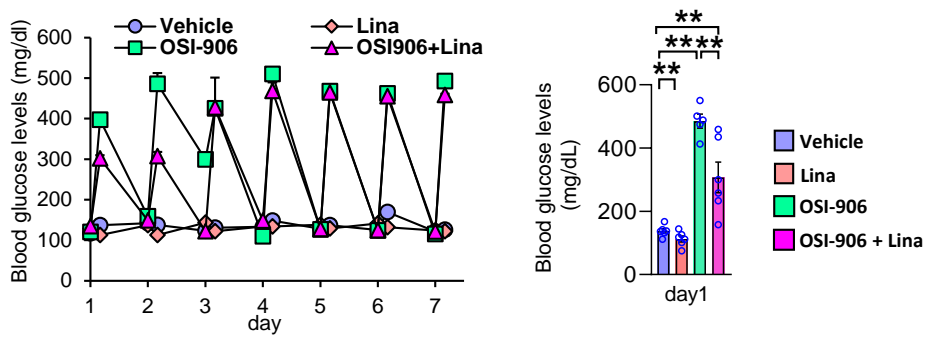

(d)

(e)

(f)

(g)
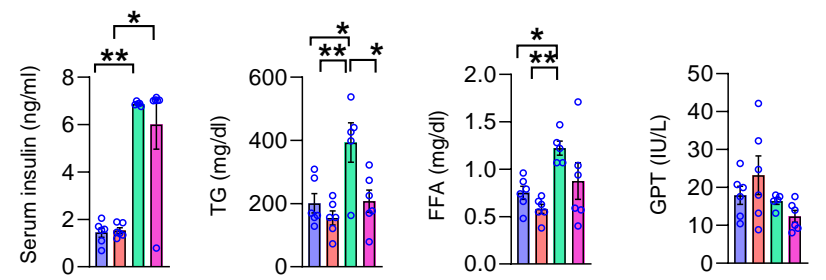

$\square$ Vehicle

$\square$ Lina

$\square$ OSI-906

OSI-906 + Lin 
Fig. 2

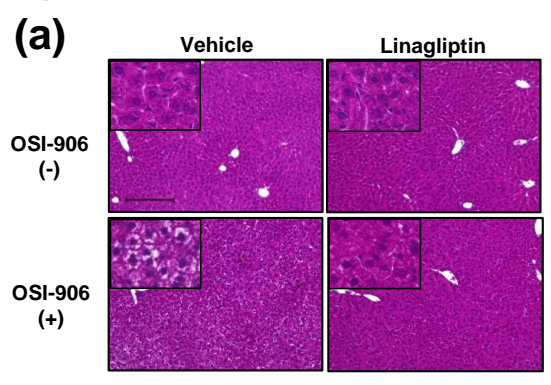

(e)
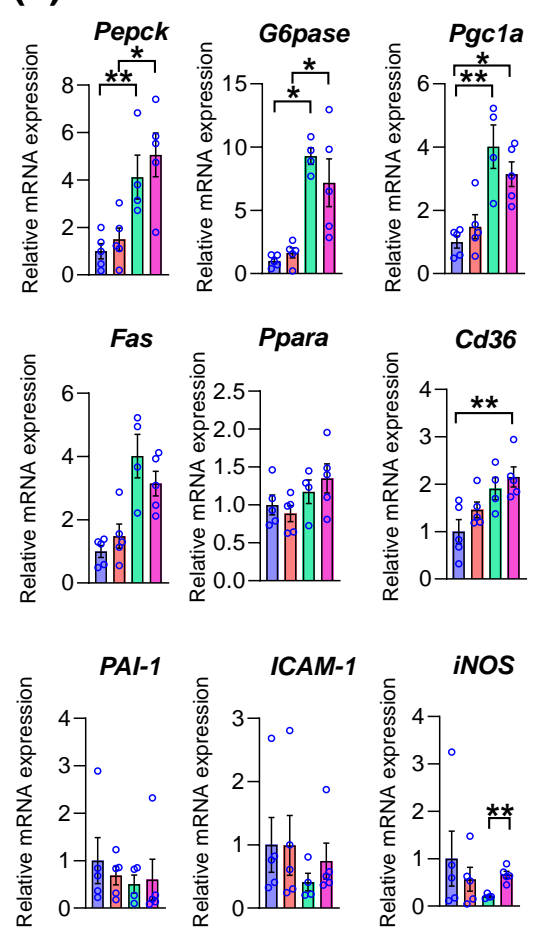

(b)

(c)

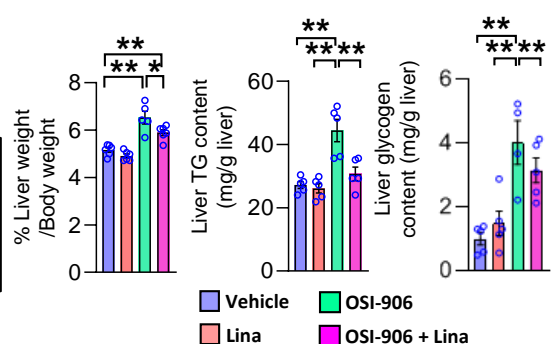

$\square$ Lina $\square$ osI-906 + Lina
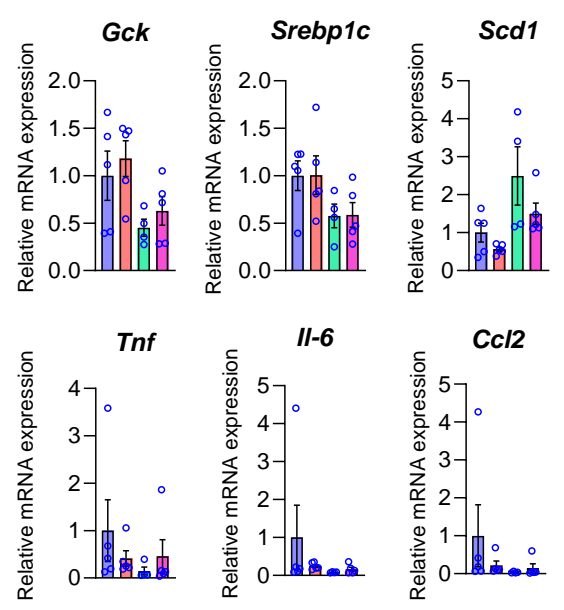

$\square$ Vehicle

$\square$ Lina

$\square$ OSI-906

$\square$ OSI-906 + Lina 


\section{Fig. 3}

(a)

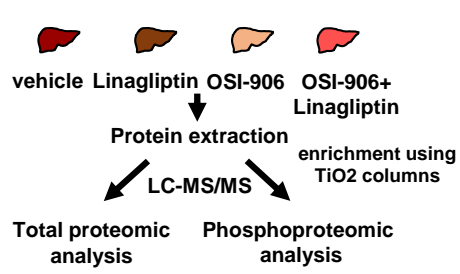

(b)
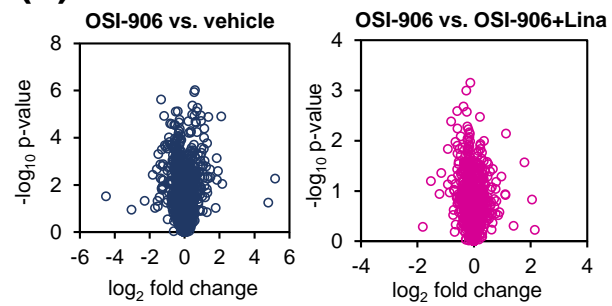

(c)

proteomics

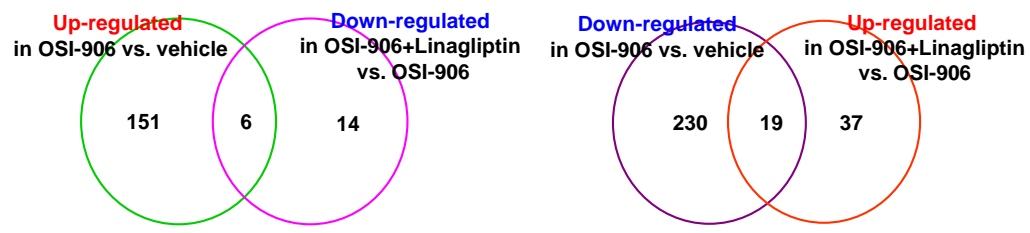




\section{Fig. 4}

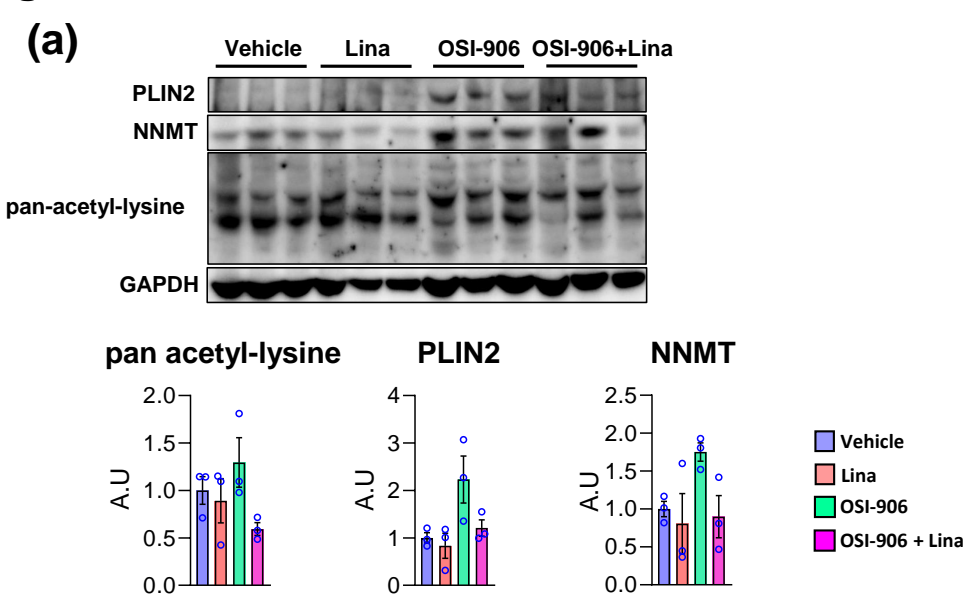

(b)
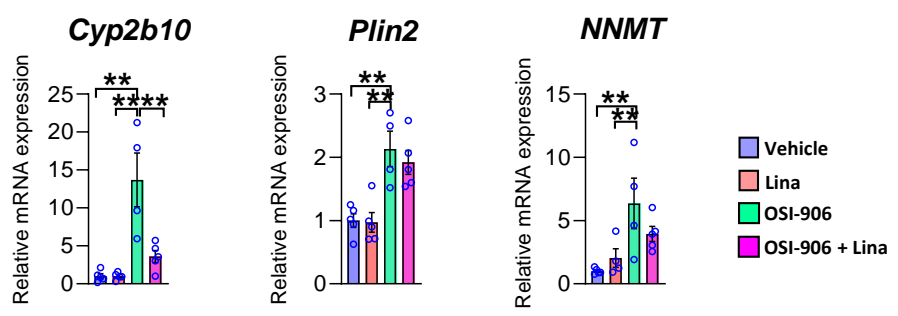


\section{Fig. 5}

\section{OSI-906 vs. vehicle}

$-\log _{10}(P$ value $)$

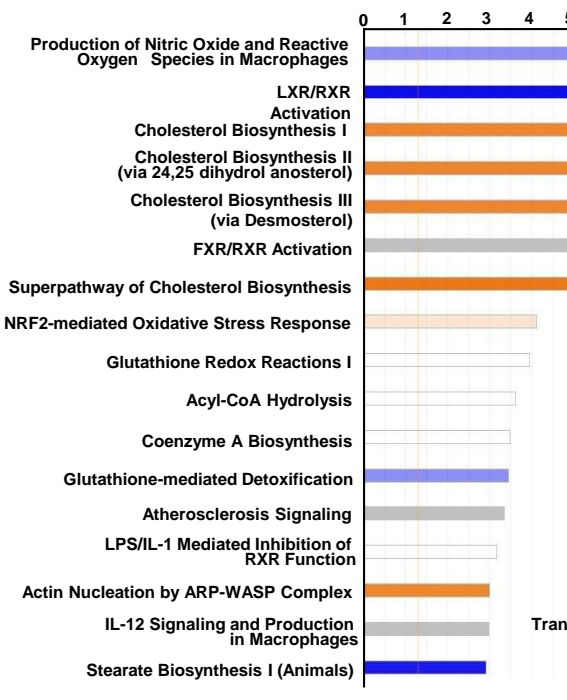

OSI-906 + Lina vs. OSI-906

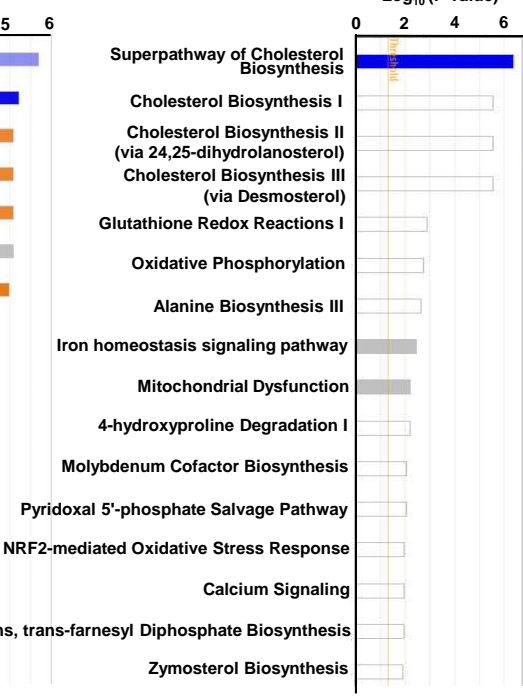


Fig. 6
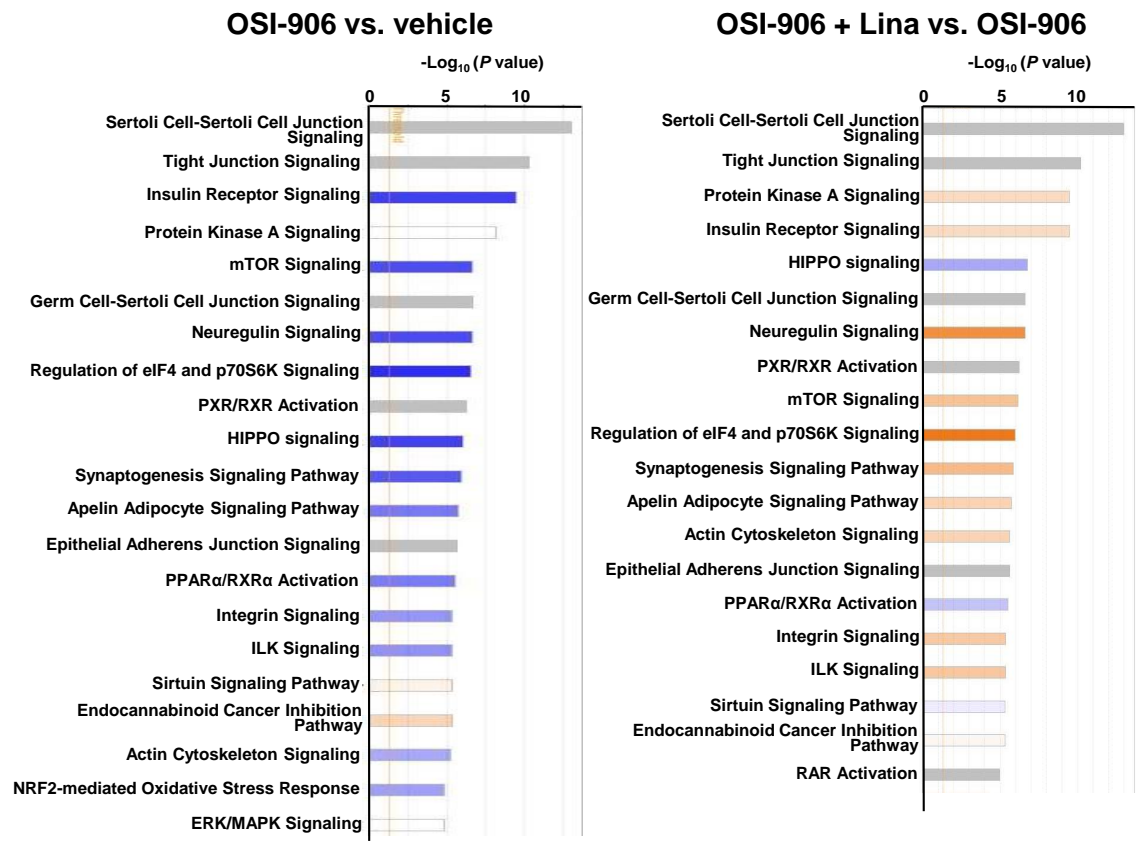
Fig. 7
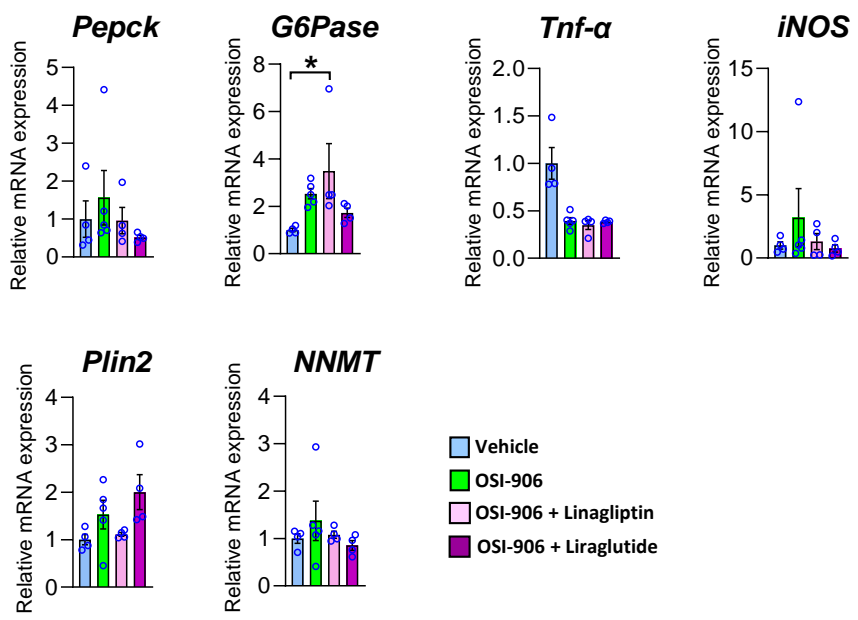

$\square$ Vehicle

$\square$ OSI-906 + Linagliptin

$\square$ oSI-906 + Liraglutide 
Fig. 8

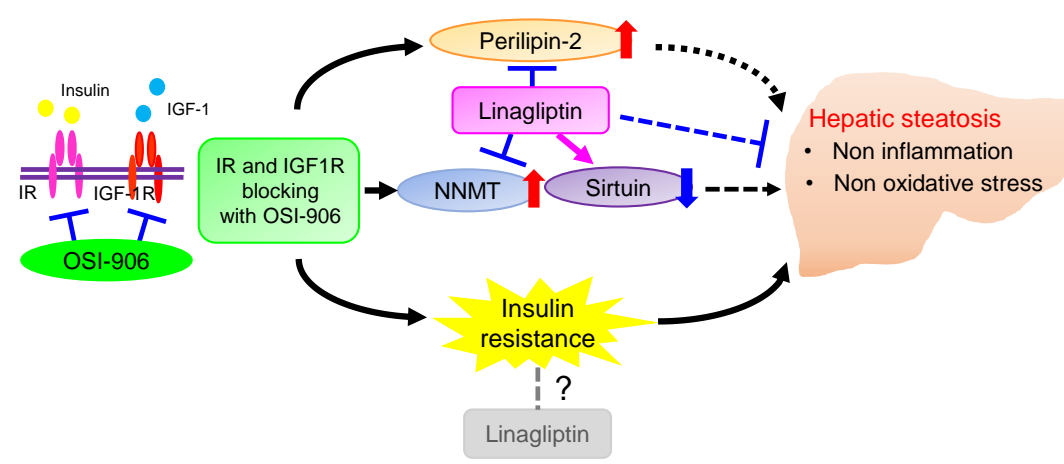

